\begin{tabular}{|c|c|c|}
\hline \multirow{3}{*}{$\begin{array}{r}\text { Case Reports in } \\
\text { Gastroenterology }\end{array}$} & \multirow{2}{*}{\multicolumn{2}{|c|}{ Case Rep Gastroenterol 2015;9:101-105 }} \\
\hline & & \\
\hline & $\begin{array}{l}\text { DOI: 10.1159/000381394 } \\
\text { Publisned online: April Z2, } 2015\end{array}$ & $\begin{array}{l}\text { ( } 2015 \text { S. Karger AG, Basel } \\
1662-0631 / 15 / 0091-0101 \$ 39.50 / 0 \\
\text { www.karger.com/crg }\end{array}$ \\
\hline & \multicolumn{2}{|c|}{$\begin{array}{l}\text { This is an Open Access article licensed under the terms of the Creative Common } \\
\text { Attribution-NonCommercial } 3.0 \text { Unported license (CC BY-NC) (www.karger.com/OA } \\
\text { license), applicable to the online version of the article only. Distribution permitted for non } \\
\text { commercial purposes only. }\end{array}$} \\
\hline
\end{tabular}

\title{
Pure Laparoscopic Hepatectomy Combined with a Pure Laparoscopic Pringle Maneuver in Patients with Severe Cirrhosis
}

\author{
Shigehito Miyagi Chikashi Nakanishi Naoki Kawagishi Takashi Kamei \\ Susumu Satomi Noriaki Ohuchi \\ Division of Transplantation, Reconstruction and Endoscopic Surgery, Tohoku University \\ Graduate School of Medicine, Sendai, Japan
}

\section{Key Words}

Pure laparoscopic hepatectomy · Liver cirrhosis · Ascites · Pringle maneuver

\begin{abstract}
Laparoscopic hepatectomy is a standard surgical procedure. However, it is difficult to perform in patients with severe cirrhosis because of fibrosis and a high risk of hemorrhage. We report our recent experience in five cases of pure laparoscopic hepatectomy combined with a pure laparoscopic Pringle maneuver in patients with severe cirrhosis. From 2012 to 2014, we performed pure laparoscopic partial hepatectomy in five patients with severe liver cirrhosis (indocyanine green retention rate at $15 \mathrm{~min}$ [ICG R15] $>30 \%$ and fibrosis stage f4). A pure laparoscopic Pringle maneuver was employed in all patients. We investigated operative time, blood loss, duration of hospitalization and the days when discharge was possible, and compared these findings with those of patients with a normal liver (ICG R15 $<10 \%$, f0) who underwent pure laparoscopic partial hepatectomy during the same period $(n=7)$. As a result, operative time, blood loss, duration of hospitalization and the days when discharge was possible were similar in patients with cirrhosis undergoing pure laparoscopic hepatectomy combined with a pure laparoscopic Pringle maneuver to those in patients with a normal liver undergoing pure laparoscopic partial hepatectomy. In conclusion, pure laparoscopic hepatectomy combined with a pure laparoscopic Pringle maneuver appears to be safe in patients with severe cirrhosis.

(c) 2015 S. Karger AG, Basel
\end{abstract}

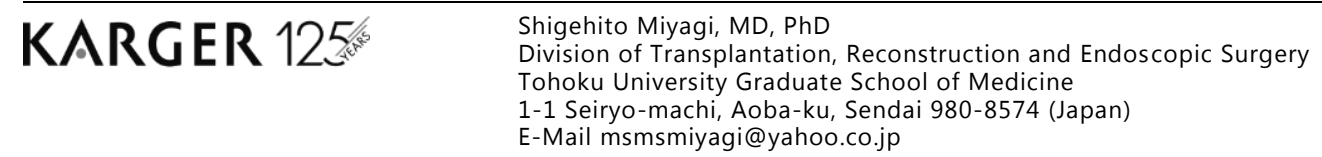


Miyagi et al:: Pure Laparoscopic Hepatectomy Combined with a Pure Laparoscopic Pringle Maneuver in Patients with Severe Cirrhosis

\section{Introduction}

Radiofrequency ablation is a standard therapy for patients with hepatic tumors and severe liver cirrhosis who cannot tolerate more extensive surgery. However, this procedure has an important limitation: no tissue specimen is obtained from the tumor for pathological evaluation. Recently, pure laparoscopic partial hepatectomy has also become an accepted therapy for patients with severe cirrhosis. Pure laparoscopic hepatectomy is a less invasive procedure than conventional open hepatectomy or laparoscopic-assisted hepatectomy [1], and it provides tissue for pathological examination. However, in patients with severe liver cirrhosis, laparoscopic surgery is difficult to perform because of liver fibrosis and a high risk of hemorrhage [2, 3].

Pure laparoscopic hepatectomy minimizes both the destruction of collateral blood flow and lymphatic flow caused by laparotomy and limits hepatic mesenchymal injury caused by compression. This is especially beneficial for patients with liver cirrhosis. Therefore, pure laparoscopic hepatectomy lowers the risk of water or electrolyte imbalance and hypoproteinemia by reducing postoperative ascites production [2, 3]. However, the safety and potential contraindications for this procedure in patients with complete liver cirrhosis are poorly defined.

Recently, we performed pure laparoscopic hepatectomy combined with a Pringle maneuver in five patients with severe cirrhosis (indocyanine green retention rate at $15 \mathrm{~min}$ [ICG R15] >30\% and fibrosis stage f4) [4]. The purpose of this study was to investigate the safety of this procedure in patients with severe liver cirrhosis.

\section{Case Report}

Between 2012 and 2014 we performed pure laparoscopic partial hepatectomy combined with a Pringle maneuver in five patients with severe cirrhosis (ICG R15 $>30 \%$, f4) [4]. The pure laparoscopic Pringle maneuver was employed in all patients to reduce hemorrhage. We investigated operative time, blood loss, duration of hospitalization, and the days when discharge was possible, and compared these findings with those of patients with a normal liver (ICG R15 <10\%, f0) who underwent pure laparoscopic partial hepatectomy during the same period $(n=7)$. The days when discharge was possible were granted when a drain and intravenous feeding were not necessary and any pain was under self-control.

Patients were observed from the date of the operation until the last follow-up appointment. All values shown are mean \pm standard deviation. Statistical analyses were conducted using the Mann-Whitney U test. All calculations were made using the JMP Pro software package (SAS Institute, Cary, N.C., USA). A p value $<0.05$ was considered statistically significant. All procedures were reviewed and approved by the Ethical Committee of Tohoku University Graduate School of Medicine and were performed in accordance with the ethical standards of the Declaration of Helsinki.

As the result, no postoperative mortality or bleeding was observed. There were no surgical complications >grade IIIb according to the Clavien-Dindo classification. The patient characteristics are presented in table 1. The ICG R15 level was significantly higher in the severe liver cirrhosis cases than in the normal liver cases, but there were no other significant differences in patient characteristics between the two groups.

The mean operative time in the severe liver cirrhosis cases $(268.4 \pm 86.5 \mathrm{~min})$ was not significantly different from that in the normal liver cases $(215.4 \pm 77.0 \mathrm{~min})$. The total mean bleeding volume was slightly higher in the severe liver cirrhosis cases $(226.0 \pm 203.8 \mathrm{ml})$ 
Miyagi et al.: Pure Laparoscopic Hepatectomy Combined with a Pure Laparoscopic Pringle Maneuver in Patients with Severe Cirrhosis

than in the normal liver cases $(46.7 \pm 62.9 \mathrm{ml})$, but the difference was not statistically significant. The total mean days of hospitalization were $7.4 \pm 0.9$ days in the severe liver cirrhosis cases and $7.0 \pm 0.8$ days in the normal liver cases; this difference was not statistically significant. The total mean days when discharge was possible were $4.0 \pm 0.5$ days in the severe liver cirrhosis cases and $4.6 \pm 0.4$ days in the normal liver cases; this difference was not statistically significant (table 2).

\section{Discussion}

Pure laparoscopic partial hepatectomy is a minimally invasive procedure that has recently become useful in patients with severe cirrhosis. The advantages of this approach over radiofrequency ablation include the acquisition of tissue for pathological examination. Therefore, we investigated the safety and efficacy of pure laparoscopic partial hepatectomy combined with a pure laparoscopic Pringle maneuver in patients with severe liver cirrhosis. We found that there were no significant differences in any of the parameters investigated between patients with cirrhosis undergoing pure laparoscopic partial hepatectomy combined with a pure laparoscopic Pringle maneuver and patients with a normal liver undergoing laparoscopic partial hepatectomy. These findings indicate that pure laparoscopic partial hepatectomy combined with a pure laparoscopic Pringle maneuver is a safe surgical procedure for patients with severe cirrhosis.

We believe that the key to this technique was the addition of the pure laparoscopic Pringle maneuver. Since this technique is performed laparoscopically, it allows the maintenance of abdominal cavity pressure. Because of this, the pure laparoscopic Pringle maneuver is an effective method in patients with severe cirrhosis for controlling not only arterial-portal bleeding, but also venous bleeding. In addition, maintaining abdominal pressure reduces lymphorrhea and ascites [5, 6]. Some investigators believe that the Pringle maneuver is not necessary, but we believe it enhances safety in emergencies, especially in patients with cirrhosis. Our study included a small number of patients; larger studies are necessary in the future.

Our study has several limitations. In this study, we investigated only cases undergoing partial resection. We must assume that pure laparoscopic hepatectomy is safe and appropriate only in patients undergoing partial resection. However, we believe that pure laparoscopic hepatectomy is also safe in patients undergoing anatomical resection. During pure laparoscopic surgery, adequate patient positioning and port arrangement facilitates partial liver resection in the region of the subphrenic space, peri-inferior vena cava or retroperitoneum by providing a good visual field. In addition, the reduced formation of postoperative adhesions makes this procedure safer in the context of repeat hepatectomy procedures. Therefore, future studies should compare the results of partial resection and anatomical resection using this technique in patients with severe cirrhosis. With further studies, we may be able to determine whether pure laparoscopic hepatectomy can be safely used for anatomical resection.

In conclusion, although pure laparoscopic partial hepatectomy for patients with severe cirrhosis may be difficult, complications were few when the Pringle laparoscopic maneuver was used. These results indicate that this combined technique is safe when used in patients with severe cirrhosis. 
Miyagi et al.: Pure Laparoscopic Hepatectomy Combined with a Pure Laparoscopic Pringle Maneuver in Patients with Severe Cirrhosis

\section{Acknowledgment}

This work was supported by Grants-in-Aid for Scientific Research from the Ministry of Education, Science and Culture of Japan and from the Ministry of Welfare of Japan, and by a grant from Tohoku University Graduate School of Medicine.

\section{Disclosure Statement}

The authors have no conflicts of interest to declare.

\section{References}

1 Kaneko H, Takagi S, Otsuka Y, Tsuchiya M, Tamura A, Katagiri T, Maeda T, Shiba T: Laparoscopic liver resection of hepatocellular carcinoma. Am J Surg 2005;189:190-194.

-2 Kaneko H, Tsuchiya M, Otsuka Y, Yajima S, Minagawa M, Watanabe M, Tamura A: Laparoscopic hepatectomy for hepatocellular carcinoma in cirrhotic patients. J Hepatobiliary Pancreat Surg 2009;16:433-438.

-3 Kanazawa A, Tsukamoto T, Shimizu S, Kodai S, Yamazoe S, Yamamoto S, Kubo S: Impact of laparoscopic liver resection for hepatocellular carcinoma with F4-liver cirrhosis. Surg Endosc 2013;27:2592-2597.

-4 Sheng QS, Lang R, He Q, Yang YJ, Zhao DF, Chen DZ: Indocyanine green clearance test and model for end-stage liver disease score of patients with liver cirrhosis. Hepatobiliary Pancreat Dis Int 2009;8:46-49.

5 Morise Z, Kawabe N, Kawase J, Tomishige H, Nagata H, Ohshima H, Arakawa S, Yoshida R, Isetani M: Pure laparoscopic hepatectomy for hepatocellular carcinoma with chronic liver disease. World J Hepatol 2013;5: 487-495.

-6 Kim SJ, Jung HK, Lee DS, Yun SS, Kim HJ: The comparison of oncologic and clinical outcomes of laparoscopic liver resection for hepatocellular carcinoma. Ann Surg Treat Res 2014;86:61-67.

Table 1. Patient characteristics

\begin{tabular}{lcc}
\hline & $\begin{array}{l}\text { Severe cirrhosis } \\
(\mathrm{n}=5)\end{array}$ & $\begin{array}{l}\text { Normal liver } \\
(\mathrm{n}=7)\end{array}$ \\
\hline Age, years & $65.2 \pm 6.3$ & $57.3 \pm 15.3$ \\
Sex ratio, M:F & $2: 3$ & $4: 3$ \\
HCC: others & $5: 0$ & $3: 4$ \\
Tumor size, mm & $22.0 \pm 8.2$ & $24.1 \pm 18.6$ \\
ICG R15 & $33.5 \pm 2.2$ & $7.5 \pm 2.7^{*}$ \\
Fibrosis stage, f0:f4 & $0: 5$ & $5: 0$ \\
Child-Pugh score, A:B & $4: 1$ & $7: 0$ \\
\hline
\end{tabular}

HCC $=$ Hepatocellular carcinoma.

$* \mathrm{p}<0.05$ vs. group I and group II. 
Miyagi et al.: Pure Laparoscopic Hepatectomy Combined with a Pure Laparoscopic Pringle Maneuver in Patients with Severe Cirrhosis

Table 2. Comparison of the two groups

\begin{tabular}{lcc}
\hline & Severe cirrhosis $(\mathrm{n}=5)$ & Normal liver $(\mathrm{n}=7)$ \\
\hline Operative time, min & $268.4 \pm 86.5$ & $215.4 \pm 77.0$ \\
Blood loss, ml & $226.0 \pm 203.8$ & $46.7 \pm 62.9$ \\
Hospitalization, days & $7.4 \pm 0.9$ & $7.0 \pm 0.8$ \\
Days when discharge was possible & $4.0 \pm 0.5$ & $4.6 \pm 0.4$ \\
\hline
\end{tabular}

